\title{
Parasitoses gastrintestinais de caprinos e ovinos comercializados na feira de animais de Tabira, Sertão de Pernambuco ${ }^{1}$
}

\author{
Clécio Henrique Limeira ${ }^{2}$; Ana Célia Rodrigues Athayde $^{3}$; José Romero Alexandre Alves ${ }^{4}$; \\ Geilson Manoel de Souza Lima; ${ }^{5}$ Manoella de Queiroz Rodrigues Limeira ${ }^{6}$; Maria do Carmo \\ de Medeiros ${ }^{7}$
}

\begin{abstract}
Resumo: No Nordeste do Brasil, muitos produtores utilizam a venda de animais vivos em feiras livres como principal via de comercialização de seus animais. Embora contribuam para o desenvolvimento econômico e social, essas feiras tornam-se facilitadoras no processo de transmissão e disseminação de doenças parasitárias. Nesse sentido, o objetivo deste trabalho foi caracterizar a ocorrência de parasitismo gastrintestinalem caprinos e ovinos comercializados na feira de animais de Tabira, estado de Pernambuco. Foram coletadas fezes e sangue de 84 caprinos e 92 ovinos, entre novembro de 2014 e janeiro de 2015. Os caprinos apresentaram frequência de 70,24\% e os ovinos 78,26\% de animais positivos na contagem de ovos por grama de fezes (OPG). As fêmeas caprinas $(75,44 \%)$ e os machos ovinos $(82,93 \%)$ adultos foram mais acometidos. Os caprinos apresentaram maior média de OPG (2.431), maior proporção de anemia $(40,68 \%)$ e maiores níveis de infecção pesada $(11,87 \%)$ e fatal (20,34\%) em comparação aos ovinos (1203; 20,86\%; 9,72\% e 15,28\%, respectivamente). Na coprocultura, larvas de Haemonchus sp. foram as mais frequentes, tanto em caprinos (90,34\%), quanto em ovinos $(91,87 \%)$. Os caprinos e ovinos comercializados na feira de animais de Tabira, estado de Pernambuco, são acometidos por grandes cargas parasitárias, sugerindo maiores cuidados dos proprietários quando da aquisição de animais nesses recintos, a fim de evitar maiores prejuízos.
\end{abstract}

Palavras-chave: Anemia; Doenças parasitárias; Haemonchus sp.; OPG; Pequenos ruminantes.

\section{Gastrointestinal parasites of caprine and ovine animals commercialized in animals fair in the city of Tabira, Sertão of Pernambuco}

\begin{abstract}
In Northeast the Brazil, many producers use the sale of live animals in free fair s as the main way of market of their animals. Although they contribute to the economic and social development, these fairs become facilitators in the process of transmission and spread of parasitic diseases. In this sense, the aim of this study was to characterize the occurrence of gastrointestinal parasitism in sheep and goats commercialized at the animals fair in Tabira, state of Pernambuco. Were collected feces and blood of 84 caprine and 92 sheep, between November 2014 and January 2015. The caprine had a frequency of 70,24\% and the ovine $78,26 \%$ of positive animals in the egg count per gram of feces (EPG). The female caprines $(75,44 \%)$ and the male ovines $(82,93 \%)$ adults were most affected. The caprine presented higher average of EPG $(6,804)$, a higher proportion of anemia $(40,68 \%)$ and higher levels of heavy infection $(11,87 \%)$ and fatal $(20,34 \%)$ compared to ovines $(1203 ; 20,86 \% ; 9,72 \%$ e $15,28 \%$, respectively). In coproculture, larvae of Haemonchu sp. are the most common, both in caprines $(90,34 \%)$ and in ovines $(91,87 \%)$. The caprines and ovines commercialized at the animals fair in Tabira, state of Pernambuco, are affected by large parasitic loads, suggesting greatercare of the owners for the acquisition of animals in enclosures in order to avoid further losses.
\end{abstract}

Keywords: Anemia; Parasitic diseases; Haemonchus sp.; EPG; Small ruminants.

\footnotetext{
${ }^{1}$ Submetido em 14/02/2017 e aprovado em 22/02/2018

${ }^{2}$ Mestre em Medicina Veterinária; Doutorando, Universidade Federal de Campina Grande (UFCG), Programa de Pós-Graduação em Medicina Veterinária, Patos-PB, CEP: 58.708-110;E-mail: cleciolimeira@hotmail.com

${ }^{3}$ Doutora em Ciências Biológicas; Professora, Universidade Federal de Campina Grande (UFCG), Programa de Pós-Graduação em Medicina Veterinária, Patos-PB, CEP: 58.708-110;E-mail: athayde@cstr.ufcg.edu.br

${ }^{4}$ Mestre em Medicina Veterinária; Doutorando, Universidade Federal de Campina Grande (UFCG), Programa de Pós-Graduação em Medicina Veterinária, Patos-PB, CEP: 58.708-110;E-mail: j.romeroalves@gmail.com

${ }^{5}$ Medico Veterinário; Técnico em Defesa Agropecuária, Agência de Defesa e Fiscalização Agropecuária de Pernambuco (ADAGRO), São José do Egito-PE, CEP: 56.700-000; E-mail: geilsonmsl@hotmail.com

${ }^{6}$ Bióloga; Dout oranda, Universidade Federal Rural de Pernambuco (UFRPE), Programa de Pós-Graduação em Botânica, Recife-PE, CEP: 52.171-900; E-mail: manoella_rodrigues@hotmail.com

${ }^{7}$ Mestre em Zootecnia; Professora, Faculdades Integradas de Patos (FIP), Patos-PB, CEP: 58.704-000; E-mail: mariacar.medeiros@hotmail.com
} 


\section{Introdução}

Embora tenha apresentado evoluções significativas nos últimos anos, a caprinovinocultura no Nordeste brasileiro apresenta gargalos que impedem a competitividade com as outras regiões do Brasil. Possuidores de um rebanho de 8,91 milhões caprinos e 11,16 milhões de ovinos, o que representa cerca de $92,7 \%$ e $60,6 \%$, respectivamente, do rebanho nacional destas espécies (IBGE, 2015), grande parte dos produtores nordestinos utilizam sistemas de produção com pouca tecnificação, predominando aqueles que têm a criação de pequenos ruminantes como uma atividade secundária, não adotando práticas sanitárias básicas como quarentena e área de isolamento, além de não receberem assistência técnica de maneira adequada (Coelho et al., 2011).

Devido a essa desorganização, muitos produtores nordestinos utilizam a venda de animais vivos em feiras livres como principal via de comercialização de seus animais (Sousa, 2007). Este tipo de comercio é muito comum no Nordeste, principalmente em cidades do interior, contribuindo para a economia local e para o desenvolvimento de muitos municípios do semiárido brasileiro (Maia, 2007). Em Tabira, estado de Pernambuco, a feira de animais é uma referência no comércio de caprinos e ovinos, tornando o município um grande fornecedor desses animais para cidades vizinhas e até outros estados.

Com relação à sanidade, entre os vários problemas enfrentados pelos produtores de caprinos e ovinos, as parasitoses gastrintestinais, cujos agentes etiológicos são espécies de nematódeos pertencentes à Superfamília Trichostrongyloidea, continua sendo o mais importante do ponto de vista sanitário dos rebanhos, e as manifestações deste parasitismo dependem de quais espécies de nematódeos estão presentes, a intensidade da infecção, a categoria e o estado geral do hospedeiro (Vieira, 2008). Oesophagostomum sp, Cooperia spp, Haemonchus sp, Trichostrongylus spp e Bunostomum sp são os membros da superfamília Trichostrongyloidea encontradas com mais frequência, sendo Haemonchus contortus o nematódeo mais abundante entre os causadores de parasitoses gastrintestinais de caprinos e ovinos, embora outras espécies possam ser mais prevalentes em alguns casos (Souza et al., 2012).

Além de ser o mais frequente, o $H$. contortus também é o parasita mais importante sob ponto de vista clinico em pequenos ruminantes, levando a quadros severos de anemia, emagrecimento, edema submandibular e diarreia (Almeida et al., 2013; Alencar et al., 2010), e embora esses sinais clínicos estejam presentes em outras enfermidades infecciosas e/ou nutricionais, são nas parasitoses gastrintestinais (principalmente nas causadas pelo $H$. contortus) que eles se tornam mais aparentes, podendo de causar mortalidade considerável (Lira et al., 2013).

Desta forma, é fundamental o conhecimento sobre a epidemiologia, espécies envolvidas e fatores socioeconômicos relacionados à transmissão e sobrevivência dos parasitos gastrintestinais de pequenos ruminantes no semiárido nordestino, para que sejam utilizadas medidas de controle de acordo com a realidade de cada região. Nesse sentido, o objetivo do presente trabalho foi caracterizar a ocorrência de parasitas gastrintestinais de caprinos e ovinos comercializados na feira de animais de Tabira, Sertão de Pernambuco, levando em consideração aspectos da dinâmica que envolve o fluxo de animais da feira.

\section{Mate rial e Métodos}

\subsection{Localização e caracte rização da área de estudo}

As coletas foram realizadas em caprinos e ovinos comercializados na feira de animais da cidade de Tabira (longitude $07^{\circ} 35^{\prime} 31^{\prime \prime} \mathrm{S}$ e latitude $37^{\circ} 32^{\prime} 24^{\prime \prime}$ W), situada na Região de Desenvolvimento (RD) do Sertão do Pajeú, estado de Pernambuco. O clima é característico de regiões semiáridas, com chuvas concentradas em um único período (3 a 5 meses) e médias anuais de 400 a $800 \mathrm{~mm}$, temperaturas elevadas durante todo o ano $\left(23\right.$ a $\left.27^{\circ} \mathrm{C}\right)$, insolação de $2.800 \mathrm{~h} / \mathrm{ano}$, umidade relativa em torno de $50 \%$ e a evaporação média anual é de $2.000 \mathrm{~mm} / \mathrm{ano}$ (Brasil, 2011).

A feira de animais ocorre semanalmente, as quartas-feiras, e recebe animais do próprio estado de Pernambuco quanto de outros estados, como Bahia e Paraíba, por exemplo, caracterizando os trânsitos intraestadual e interestadual, 
respectivamente. As principais espécies comercializadas na feira são bovinos, caprinos e ovinos, embora também ocorra o comércio de suínos e aves.

\subsection{Amostragem}

Para o cálculo do número de caprinos e/ou ovinos amostrados, foram considerados os seguintes parâmetros: prevalência esperada; erro absoluto; e nível de confiança. O cálculo foi realizado utilizando a fórmula para amostras simples aleatórias (Thrusfield, 2004):

$$
n=\frac{Z^{2} x p(1-p)}{d^{2}}
$$

em que:

$\mathrm{n}=$ número de animais amostrados;

$Z=1,96$ (nível de confiança de $95 \%$ );

$p=$ prevalência esperada (de acordo com literatura);

$d=$ erro absoluto de $10 \%$ (precisão absoluta desejada).

Para a prevalência esperada foram utilizados como referência os dados de Lima (2007), que observou $68,1 \%$ de caprinos e $59 \%$ de ovinos positivos para parasitos gastrintestinais na região do sertão do Pajeú, estado de Pernambuco. Desta forma foram coletadas amostras de 84 caprinos e 92 ovinos, durante o período de outubro de 2014 a janeiro de 2015, separando-os em quatro categorias por espécie: machos com menos de seis meses, machos com mais de seis meses, fêmeas com menos de seis meses e fêmeas com mais de seis meses de idade, além de dividi-los em dois outros grupos, intraestadual e interestadual, de acordo com a localidade de origem dos mesmos. Dos 84 caprinos utilizados, 59 tinham como origem o estado de Pernambuco e 25 a Bahia, e dos 92 ovinos, 66 foram provenientes de Pernambuco e 26 da Bahia.

A escolha dos proprietários que participaram da pesquisa foi feita através de amostra por conveniência (Thrusfield, 2004). Ao todo, seis proprietários, designados pela sequência das letras de $\mathrm{A}$ à $\mathrm{F}$, concordaram em participar, assinando o respectivo Termo de Consentimento Livre e Esclarecido e os animais eram selecionados aleatoriamente, entre aqueles presentes no curral correspondente a cada proprietário

\subsection{Coleta das amostras e exames laboratoriais}

As coletas de fezes foram realizadas manualmente, diretamente da ampola retal dos animais, utilizando-se luvas plásticas que eram invertidas posteriormente, servindo como recipiente primário para o transporte. Também foi coletado de cada animal $5 \mathrm{ml}$ de sangue por punção da veia jugular externa, utilizando tubos à vácuo com anticoagulante (EDTA 10\%). Tanto as amostras de fezes quanto as de sangue foram identificadas com a letra correspondente a cada proprietário, seguida do número sequencial de coleta do dia. Em seguida as amostras foram acondicionadas em caixas de isopor contendo gelo e transportadas até o Laboratório de Doenças Parasitarias dos Animais Domésticos (LAPAD), da Universidade Federal de Campina Grande (UFCG), Patos - PB, onde as amostras de fezes foram processadas. Já o sangue contendo EDTA $10 \%$ foi levado até um laboratório particular, também na cidade de Patos-PB, para obtenção do valor do hematócrito de cada animal, segundo técnica descrita por Jain (1993). De acordo as recomendações de Benesi (1985), animais com hematócrito superiores a $25 \%$ foram classificados sem anemia, e com anemia os que apresentaram hematócrito menores que esse valor, não sendo adotado, neste trabalho, o grau de anemia proposto pelo mesmo.

Com as amostras de fezes, foi determinado o número de ovos por grama de fezes (OPG) pelo método de Gordon e Whitlock (1939). A coprocultura seguiu o método descrito por Roberts e O'Sullivan (1959), utilizando-se um pool das amostras por proprietário, e a identificação dos gêneros de larvas de terceiro estádio (L3) foi realizada por meio das descrições de Ueno e Gonçalves (1998). Após a determinação da contagem de OPG, os animais positivos nesse teste foram classificados de acordo com o grau de infecção em quatro níveis, usando como referência a classificação descrita por Ueno e Gonçalves (1998): infecção leve (OPG <500), moderada (OPG $500-1500$ ), pesada (OPG 1501 - 3000) e fatal $(\mathrm{OPG}>3000)$.

\subsection{Análises estatísticas dos dados}

Foi realizada análise estatística descritiva por meio de distribuições absolutas e percentuais (Thrusfield, 2004), para a identificação da frequência de parasitos gastrintestinais de caprinos e ovinos.

Também foram realizadas técnicas de estatística inferencial, seguindo sugestões de Ayres et al. (2007). Previamente a estas análises, os dados foram submetidos ao Teste de Lilliefors 
para verificar sua distribuição, sendo observado que os números de OPG não apresentam distribuição normal, utilizando-se, portanto, testes não paramétricos para comparação das médias. As médias de OPG de caprinos e ovinos foram comparadas como amostras independentes, por meio do Teste U de Mann-Whitney. Este teste também foi usado para verificar se existe diferença estatística entre média de OPG de animais de origem intraestadual e interestadual comercializados na feira. Já para a comparaçãodo OPG entre as quatro categorias, foi utilizado o Teste de Kruskal-Wallis para amostras não paramétricas. A análise descritiva de associação entre os valores de hematócrito e OPG foram submetidas à correlação de Spearmann e consideradas significativas quando $\mathrm{p}<0,05$.

Para a decisão de todos os testes estatísticos, foi observado o nível de significância de 5\%. O programa utilizado para a obtenção da análise estatística foi o BioEstat versão 5.3 (Ayres et al., 2007).

\subsection{Procedimentos éticos}

O projeto foi apreciado e aprovado pelo Comitê de Ética em Pesquisa (CEP), do Centro de Saúde e Tecnologia Rural - CSTR/UFCG, e protocolado sob o número 213/2014

\section{Resultados}

A Tabela 1 demonstra o número geral de animais utilizados, cidade de origem, frequência de animais positivos e a média de OPG. Os animais dos proprietários $\mathrm{A}$ e $\mathrm{C}$ eram provenientes do estado da Bahia, enquanto os outros proprietários $(\mathrm{B}, \mathrm{D}, \mathrm{E}$ e F) trouxeram seus animais do próprio estado de Pernambuco.

Tabela 1 Quantitativo geral e origem de caprinos e ovinos utilizados para coleta de amostras, com frequência de animais positivos e média de OPG, por proprietário

\begin{tabular}{cccccc}
\hline Proprietário & Origem & Espécie & $\mathbf{N}^{\mathbf{0}}$ animais & $\mathbf{N}^{\mathbf{0}}$ animais positivos (\%) & Média OPG \\
\hline A & Casa Nova/BA & Caprino & 25 & $21(84)$ & 6804 \\
B & Tabira/PE & Ovino & 22 & $14(63,64)$ & 1723 \\
C & Remanso/BA & Ovino & 26 & $21(80,77)$ & 1627 \\
D & Solidão/PE & Ovino & 19 & $16(84,21)$ & 547 \\
E & Tabira/PE & Ovino & 19 & $16(84,21)$ & 1058 \\
F & Tabira/PE & Caprino & 59 & $38(64,41)$ & 502 \\
& & Ovino & 6 & $5(83,34)$ & 317 \\
\hline
\end{tabular}

Com relação aos caprinos, as maiores frequências de positivos e média de OPG nos exames parasitológicos foram observadas nos animais de origem na Bahia (84\% e 6804 OPG), enquanto os animais provenientes de Pernambuco apresentaram frequência de $64,41 \%$ e média de 502 OPG. Já entre os ovinos, os de origem em Pernambucoapresentaramas maiores frequências de positivos $(84,21 \%)$ e também média de OPG
(1723), em comparação com os de origem na Bahia $(80,77 \%$ e 1627 OPG).

Os ovinos $(78,26 \%)$ apresentaram uma frequência de animais positivos maior em relação aos caprinos $(70,24 \%)$, embora estes últimos tenham apresentado uma maior média de OPG (2.431) emcomparaçãocom os primeiros (1.203), com significância estatística $(\mathrm{p}>0,05)$ no teste de Mann-Whitney (Tabela 2).

Tabela 2 Frequência de animais positivos e média de OPG de caprinos e ovinos da feira de animais de Tabira/PE, considerando o sexo, idade e origem dos animais

\begin{tabular}{|c|c|c|c|c|c|c|c|}
\hline \multirow[b]{2}{*}{ Variáveis } & \multirow[b]{2}{*}{ Categoria } & \multicolumn{3}{|c|}{ Caprinos } & \multicolumn{3}{|c|}{ Ovinos } \\
\hline & & $\begin{array}{c}\mathrm{N}^{0} \text { de } \\
\text { animais }\end{array}$ & $\begin{array}{c}\text { Animais } \\
\text { positivos }(\%)\end{array}$ & $\begin{array}{l}\text { Média } \\
\text { OPG }\end{array}$ & $\begin{array}{c}\mathrm{N}^{0} \text { de } \\
\text { animais }\end{array}$ & $\begin{array}{c}\text { Animais } \\
\text { positivos }(\%)\end{array}$ & $\begin{array}{l}\text { Média } \\
\text { OPG }\end{array}$ \\
\hline \multirow{2}{*}{ Sexo } & Macho & 27 & $16(59,26)$ & 1211 & 41 & $34(82,93)$ & 1239 \\
\hline & Fêmea & 57 & $43(75,44)$ & 2952 & 51 & $38(74,51)$ & 1212 \\
\hline \multirow{2}{*}{ Idade } & $<6$ meses & 15 & $8(53,33)$ & 3380 & 18 & $12(66,67)$ & 1111 \\
\hline & $>6$ meses & 69 & $51(73,91)$ & 2178 & 74 & $60(81,08)$ & 1251 \\
\hline \multirow{2}{*}{ Origem } & Intraestadual & 59 & $38(64,41)$ & $524^{\mathrm{a}}$ & 66 & $51(77,27)$ & $1065^{\mathrm{A}}$ \\
\hline & Interestadual & 25 & $21(84)$ & $6804^{\mathrm{A}}$ & 26 & $21(80,77)$ & $1627^{\mathrm{A}}$ \\
\hline Total & & 84 & $59(70,24)$ & $2431^{\mathrm{B}}$ & 92 & $72(78,26)$ & $1203^{b}$ \\
\hline
\end{tabular}

Letras maiúsculas e minúsculas na linha $(\mathrm{B}, \mathrm{b})$ e nas colunas $(\mathrm{A}, \mathrm{a})$ representam a diferença estatística entre as categorias analisadas, por meio do Teste U de Mann-Whitney. 
Com relação às variáveis sexo e idade, as fêmeas caprinas e os machos ovinos apresentaram maior frequência de animais positivos $(75,44 \%$ e $82,93 \%$, respectivamente), enquanto que os animais com mais de seis meses de idade apresentaram maior frequência em comparação com os jovens, tanto na espécie caprina $(73,91 \%)$ quanto na ovina $(81,01 \%)$. Embora tenham sido observadas essas diferenças na frequência, quando comparada à média de $\mathrm{OPG}$ das quatro categorias (machos e fêmeas, com mais ou com menos de seis meses) por meio do teste de Kruskal-Wallis, não foi observada diferença estatística para nenhuma das espécies $(\mathrm{p}>0,05)$ (Tabela 2).
Observa-se que tanto os caprinos $(84 \%)$ quanto os ovinos $(80,77 \%)$ com origem interestadual apresentam maior frequência que os de origem intraestadual, porém apenas entre os caprinos ocorreu diferença estatística entre as médias de OPG nessa variável quando comparadas por meio do teste de Mann-Whitney. $(\mathrm{p}<0,001)$ (Tabela 2).

Os caprinos apresentaram um nível de infecção por ovos da Superfamília Trichostrongyloidea mais severo que os ovinos, com $11,87 \%$ dos caprinos apresentando infecção pesada e $20,34 \%$ com infecção fatal, enquanto os ovinos apresentaram $9,72 \%$ com infecção pesada e 15,28\% com infecção fatal (Tabela 3).

Tabela 3 Frequência de caprinos e ovinos positivos nas análises coproparasitológicas e com anemia de acordo com o nível de infecção por ovos de Trichostrongyloidea

\begin{tabular}{|c|c|c|c|c|c|c|}
\hline \multirow{2}{*}{\multicolumn{2}{|c|}{ Nível de infecção }} & \multirow{2}{*}{ OPG } & \multicolumn{2}{|c|}{ Caprinos } & \multicolumn{2}{|c|}{ Ovinos } \\
\hline & & & Frequência (\%) & Anemia (\%) & Frequência (\%) & Anemia $(\%)$ \\
\hline \multirow[t]{2}{*}{ Negativo } & & 0 & $25(29,76)$ & $6(24)$ & $20(21,74)$ & $3(15,00)$ \\
\hline & Leve & $<500$ & $23(38,98)$ & $3(13,04)$ & $25(34,72)$ & $3(12,00)$ \\
\hline \multirow{3}{*}{ Positivos* } & Moderada & $500-1500$ & $17(28,81)$ & $9(52,94)$ & $29(40,28)$ & $4(13,79)$ \\
\hline & Pesada & $1501-3000$ & $7(11,87)$ & $4(57,14)$ & $7(9,72)$ & $3(42,85)$ \\
\hline & Fatal & $>3000$ & $12(20,34)$ & $8(66,67)$ & $11(15,28)$ & $5(45,46)$ \\
\hline Total Positivos & & & $59(70,24)$ & $24(40,68)$ & $72(78,26)$ & $15(20,83)$ \\
\hline
\end{tabular}

*Adaptado de Ueno e Gonçalves (1998).

Tanto caprinos quanto ovinos com maior nível de infecção (pesada e fatal) apresentam maior frequência de anemia, demonstrando ser esse sinal clinico mais evidente nos animais com cargas parasitárias intensas. Nos caprinos com infecção pesada, $57,14 \%$ apresentaram anemia, enquanto os com infecção fatal $66,67 \%$ demonstraram algum grau de anemia. Já nos ovinos, a anemia ocorreu em $42,85 \%$ dos animais com infecção pesada e 45,46\% com nível fatal de

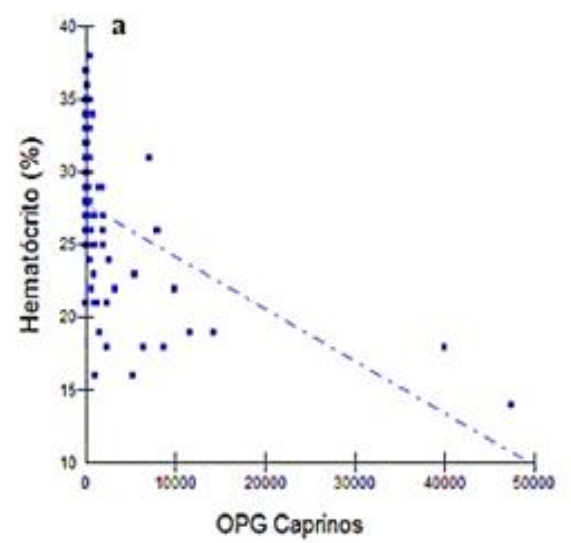

infecção (Tabela 3). Essa informação também pode ser constatada pela análise de correlação realizada entre OPG e o hematócrito, realizada através do teste de correlação de Spearman. De acordo com o teste, existe uma correlação significativa negativa para os caprinos ( $\mathrm{rs}=$ $0,5681 ; \mathrm{p}<0,001)$ e para os ovinos $(\mathrm{rs}=-0,2567$; $\mathrm{p}=0,0135$ ), sugerindo uma relação inversa entre as variáveis, ou seja, quando o número de $\mathrm{OPG}$ aumenta, o hematócrito diminui (Figura 1).

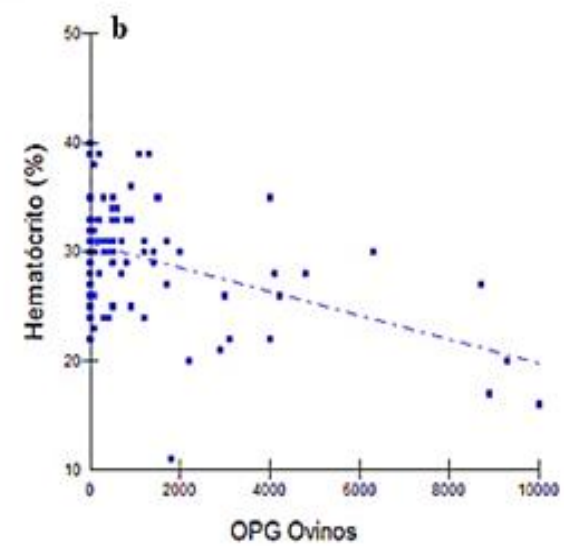

Figura 1 Dispersão demonstrando a relação inversa entre o hematócrito e OPG de caprinos (a) e ovinos (b) comercializados na feira de animais de Tabira/PE ( $r s=-0,5681$, caprinos; $r s=-0,2567$, ovinos). 
Com relação às larvas infectantes recuperadas na coprocultura, Haemonchus $s p$ foi o parasita mais frequente em caprinos $(90,34 \%)$, seguido de Trichostrongylus spp (5,52\%), Strongyloides sp $(2.76 \%)$ e Oesophagostomum $s p(1,38 \%)$. Em ovinos, também foi observado larvas de Haemonchus sp $(91,87 \%)$, Trichostrongylus spp (5,74\%), Oesophagostomum sp $(2,15 \%)$ e Strongyloides sp. $(0,24 \%)$.

\section{Discussão}

Neste trabalho, foi observado que os ovinos apresentaram maior frequência de animais positivos para ovos da Superfamília Trichostrongyloidea $(78,26 \%)$ em comparação com os caprinos $(70,24 \%)$. Dois estudos no estado da Paraíba no ano de 2014 demonstraram números parecidos, porém com os caprinos sendo a espécie mais acometida: no primeiro trabalho os pesquisadores verificaram que $79,3 \%$ dos caprinos foram positivos nas análises de OPG (Vieira et al., 2014a) e no segundo, Vieira et al. (2014b) encontraram $75.9 \%$ de ovinos positivos nos exames parasitológicos. Já Ahid et al. (2008), em região semiárida do estado do Rio Grande do Norte, também encontraram maior frequência em ovinos, observando $60,5 \%$ e $49,5 \%$ de ovinos e caprinos positivos, respectivamente. Segundo Costa et al. (2011), a frequência de parasitoses em pequenos ruminantes, depende, entre outros fatores, do habito alimentar das espécies. Quando criados de forma extensiva, em áreas nativas do semiárido, por exemplo, os ovinos tendem a ser mais susceptíveis que os caprinos, e quando em sistema intensivo ou em pastagens cultivadas, os caprinos apresentam maior susceptibilidade às parasitoses, devido a sua baixa habilidade de criar uma resposta imune contra nematódeos.

Embora tenham apresentadomenor frequência de animais positivos, foram nos caprinos que ocorreram os maiores níveis de infecção classificadas como pesada (OPG entre 1501 a 3000) e fatal (OPG >3000), além de uma maior proporção de animais anêmicos, quando analisadas as médias de OPG e a ocorrência de anemia. Segundo Molento et al. (2004), esse fato pode estar associado a maior capacidade de alguns animais suportar altas cargas parasitárias sem demonstrarem alterações clinicas, em comparação com outros. Esse processo também pode ser confirmado ao se comparar os casos clínicos de parasitoses gastrintestinais entre as duas espécies. Na Paraíba, por exemplo, Lira et al. (2013) observaram que das helmintoses gastrintestinais diagnosticadas entre 2000 e 2011, $70,9 \%$ acometiam caprinos e $29,1 \%$ os ovinos, sugerindo que os caprinos possuem maior chance de apresentar sintomatologia decorrentes das parasitoses.

Não foi verificada diferença estatística ao observar as médias de OPG das categorias animais. Porém foi observando que fêmeas caprinas e machos ovinos, ambos adultos, foram mais afetados, com $75,44 \%$ e $82,93 \%$ de animais positivos, respectivamente. Em estudo sobre fatores de risco associados à helmintoses de caprinos no Sertão da Paraíba, Vieira et al. (2014a) verificaram que as fêmeas $(81,7 \%)$ e os animais com mais de 36 meses de idade $(81,7 \%)$ foram os mais acometidos. Já Vieira et al. (2014b) observaram que os machos ovinos são mais acometidos $(83,2 \%)$, corrobando os dados obtidos neste trabalho.

Os fatos de os caprinos de origem interestadual apresentarem maiores frequência de positivos e média de OPGem comparaçãocom os de origem intraestadual, pode estar relacionado ao maior estresse submetido àqueles animais durante o transporte até a feira, já que o proprietário relatou que os animais estavamem jejum cerca de 72 horas antes de chegarem ao recinto, além da longa viagem desde a origem. Segundo Lima e Filho (2013), o estresse no transporte por longas distâncias, associado à superlotação no veículo de transporte e a não observação de parada para descanso e reidratação, causa uma fragilidade no sistema imunológico e consequentemente, maior susceptibilidade a doenças.

A correlação entre OPG e hematócrito ( $\mathrm{rs}=$ 0,5681 para caprinos e rs $=-0,2567$ para ovinos) neste estudo foi significativa e inversa, evidenciando a importância da análise desses parâmetros na identificação de animais susceptíveis às parasitoses. Resultados semelhantes foram observados por Vieira et al. (2014b) em ovinos no semiárido Paraibano, com correlação - 0,288, e em caprinos, também no estado da Paraíba, Vilela et al. (2012) verificaram correlação moderadamente negativa $(-0.292)$.

Haemonchus sp foi o parasita mais frequente entre as larvas recuperadas na coprocultura, tanto em caprinos quanto nos ovinos. Em resultados semelhantes no semiárido paraibano, Vieira et al. 
(2014b) também encontraram Haemonchus sp. como principal parasita de ovinos, representando $79.9 \%$ do total de helmintos recuperados na coprocultura, seguido de Strongyloides $s p$. (9.6\%), Trichostrongylus spp. (8.6\%), Oesophagostomum sp. (1.9\%), e Cooperia spp. $(0.1 \%)$. Já Vilela et al. (2012) encontraram Haemonchus $s p$. (80.1\%), Trichostrongylus $s p$. $(13.2 \%)$ e Oesophagostomum sp. (6.7\%) parasitando caprinos em região semiárida no Nordeste do Brasil.

A alta prevalência de Haemonchus sp na coprocultura e os níveis de infecção encontrados na contagem de ovos por grama de fezes sugere ser este parasita o responsável pelos índices de anemia observados neste trabalho. Muitos autores apontam este mesmo nematódeo como o maior causador de casos clínicos de parasitismo gastrintestinal em caprinos e ovinos em várias regiões do Brasil. No Mato Grosso do Sul, por exemplo, Almeida et al. (2013) demonstraram que a hemoncose é a doença que mais acomete ovinos, liderando o ranking de enfermidades diagnosticadas entre os anos de 1996 a 2010, com os animais apresentando sinais de apatia, emagrecimento, palidez de mucosas, edema submandibular, entre outros característicos da doença. Na Paraiba, Lira et al. (2013) também observaram que o Haemonchus sp foiresponsável pelo maior número de casos clínicos de helmintíases gastrintestinais de caprinos e ovinos no período de 2000 a 2011, com sinais de palidez de mucosas e edema submandibular presentes na maioria dos casos.

\section{Conclusão}

Os caprinos e ovinos comercializados na feira de animais de Tabira/PE são acometidos por grandes cargas parasitárias, sendo os caprinos com origem interestadual os mais acometidos. Ao adquirir animais nesse tipo de recinto, os proprietários podem estar introduzindo parasitoses gastrintestinais em seus rebanhos, causando perdas econômicas devido à diminuição de peso, retardo no crescimento, mortalidade de animais e a gastos excessivos com a compra de anti-helmínticos. Por isso é essencial que os proprietários, ao adquirirem animais nestes locais, utilizem práticas sanitárias básicas, como quarentena e tratamento seletivo, para evitar maior contaminação de suas propriedades por parasitas gastrintestinais.

\section{Agradecimentos}

Ao Conselho Nacional de Desenvolvimento Científico e Tecnológico (CNPq), pela concessão de bolsa de estudo e suporte financeiro necessário ao desenvolvimento projeto.

\section{Referências}

Ahid, S. M. M.; Suassuna, A. C. D.; Maia, M. B.; Costa, V. M. M.; Soares, H. S. Parasitas gastrintestinais de caprinos e ovinos da região oeste do Rio Grande do Norte, Brasil. Ciência Animal Brasile ira, v.9, n. 1, p.212-218, 2008. www.revistas.ufg.br/vet/article/view/3681

Alencar, S. P.; Mota, R. A.; Coelho, M. C. O. C.; Nascimento, S. A.; Abreu, S. R. O.; Castro, R. S. Perfil sanitário dos rebanhos caprinos e ovinos no Sertão de Pernambuco. Ciência Animal Brasileira, v.1, n.1, p.131-140, 2010. www.revistas.ufg.br/vet/article/view/4051

Almeida, T. L.; Brum, K. B.; Lemos, R. A. A.; Leal, C. R. B.; Borges, F. A. Doenças de ovinos diagnosticadas no Laboratório de Anatomia Patológica Animal da Universidade Federal de Mato Grosso do Sul (1996-2010). Pesquisa Veterinária Brasileira, v.33, n.1, p. 21-29, $2013 . \quad$ http://dx.doi.org/10.1590/S0100736X2013000100005

Ayres M.; Ayres Junior, M.; Ayres, D. L.; Santos, A. S. 2007. Bioestat 5.0. BIOESTAT Aplicações estatísticas nas áreas das ciências biomédicas [online]. Instituto Mamirauá. Belém, PA. 2007. Disponível em: < http://mamiraua.org.br/pt-br/downloads/programas/ $>$. Acesso em: 21 de novembro de 2014.

Benesi, F. J. Diagnóstico e terapia das anemias em caprinos. In: D’Angelino, J. L. Manejo, patologia e clínica de caprinos. São Paulo: Sociedade Paulista de Medicina Veterinária. 1985. p. 171-191.

Brasil. Ministério do Desenvolvimento Agrário. Plano Territorial de Desenvolvimento Rural Sustentável do Sertão do Pajeú. Brasilia, DF. 2011. Disponível em: < http://sit.mda.gov.br/download/ptdrs/ptdrs qua territorio 082.pdf >. Acesso em: 03 de julho de 2014.

Coelho, M. C. S. C.; Souza, V. C. de; Coelho, M. I. S.; Cunha, M. P. da; Medina, F. T. Aspectos sanitários de rebanhos caprinos e ovinos criados em assentamentos no município de PetrolinaPE. Revista Se miárido de Visu, v.1, n.1, p.32- 
40, 2011. $\quad$ http://periodicos.ifsertaope.edu.br/ojs $2 /$ index.php/revista/article/view/26

Costa, V. M. M; Simões, S. V. D; Riet-Correa, F. Controle das parasitoses gastrintestinais em ovinos e caprinos na região semiárida do Nordeste do Brasil. Pesquisa Veterinária Brasileira, v.31, n.1, p. 65-71, 2011. http://dx.doi.org/10.1590/S0100-736X2011000100010

Gordon, N. M.; Withlock, H. V. A new technique for counting nematode eggs in sheep faeces. Journal of the Council for Scientific and Industrial Research, v.12, n.1, p.50-52, 1939. http://publications.csiro.au/rpr/download?pid=procite 212 59a33-8a8e-4add-9315-f8338091a3e6\&dsid=DS1

IBGE. Diretoria de Pesquisas, Coordenação de Agropecuária. Pesquisa Pecuária Municipal. Rio de Janeiro, v.43, p.1-49, 2015. Disponível em: < http://biblioteca.ibge.gov.br/ visualizacao/periodicos/84/ppm_2015_v43_br.pdf >. Acesso em: 05 de maio de 2017.

Jain, N. C. Essentials Veterinary Hematology. Philadelphia: Lea and Febingre, 1993. 417p.

Lima, M. M. Estudo de fatores do aspecto sanitário em re lação à infe cção por parasitos gastrintestinais em caprinos e ovinos no es tado de Pernambuco - Brasil. 2007. $182 \mathrm{f}$. Tese (Doutorado em Ciência Veterinária) Universidade Federal Rural de Pernambuco, Recife, 2007.

Lima, L. R.; Barbosa Filho, J. A. D. Impacto do manejo pré-abate no bem-estar de caprinos e ovinos. Journal of Animal Behaviour and Biometeorology, v.1, n.2, p. 52-60, 2013. http://agris.fao.org/agris-

search/search.do?recordID $=$ BR2013D00006

Lira, M. A. A. de; Simões, S. V. D.; Riet-Correa, F.; Pessoa, C. M. R.; Dantas, A. F. M.; Neto, E. G. M. Doenças do sistema digestório de caprinos e ovinos no semiárido do Brasil. Pesquisa Veterinaria B rasileira, v.33, n.2, p.193-198, 2013. http://dx.doi.org/10.1590/S0100$\underline{736 \times 2013000200010}$

Maia, D. S. A feira de gado na cidade: encontros, conversas e negócios. Revista Formação, v.1, n.14, p.12-30, 2007. http://revista.fct.unesp.br/index.php/formacao/article/vie $\underline{\mathrm{w} / 695}$

Molento, M. B.; Tasca, C.; Gallo, A.; Ferreira, M.; Bononi, R.; Stecca, E. Método Famacha como parâmetro clínico individual de infecção por Haemonchus contortus em pequenos ruminantes. Ciência Rural, v.34, n.4, p.11391145, 2004. http://dx.doi.org/10.1590/S010384782004000400027

Roberts, F. H. S; O’Sullivan, J. P. Methods for egg counts and larval cultures for strongyles infesting the gastrointestinal tract of cattle. Aus tralian Journal of Agricultural Research, v.1, n.1, p.99-102, 1950. https://doi.org/10.1071/AR9500099

Sousa, W. H. O Agronegócio da caprinocultura de corte no Brasil. Revista Tecnologia \& Ciência Agropecuária, v.1, n.1, p.51-58, 2007. http://sheepembry o.com.br/files/pdf/436.pdf

Souza, M. F. de; Pimente-Neto, M.; Silva, R. M. da; Farias, A. C. B.; Guimarães, M. P. Gastrointestinal parasites of sheep, municipality of Lajes, Rio Grande do Norte. Revista B rasileira de Paras itologia Veterinária, v.21, n.1, p.71-73, 2012. http://dx.doi.org/10.1590/S1984$\underline{29612012000100015}$

Thrusfield, M. Vete rinary Epidemiology. $3^{\mathrm{a}} \mathrm{ed}$. Oxford: Blackwell Science, 2007. 624p.

Ueno, H; Gonçalves, P. C. Manual para diagnóstico das helmintoses de ruminantes. 4. ed. Tokyo: Japan International Cooperation Agency, 1998. 149p.

Vieira, L. S. Métodos alternativos de controle de nematóides gastrintestinais em caprinos e ovinos. Revista Tecnologia \& Ciência Agropecuária, v.2, n.2, p.49-56, 2008. http://revistatca.pb.gov.br/edicoes/volume-022008/volume-2-numero-2-junho-2008/tca09_metodos.pdf

Vieira, V. D.; Feitosa, T. F; Vilela, V. L. R; Azevedo, S. S; Almeida Neto, J. L. de; Morais, D. F. de; Ribeiro, A. R; Athayde, A. C. R. Prevalence and risk factors associated with goat gastrointestinal helminthiasis in the Sertão region of Paraiba State, Brazil. Tropical Animal Health and Production, v.46, n.2, p.355-61, 2014a. https://www.ncbi.nlm.nih.gov/pubmed/24214525

Vieira, V. D.; Vilela, V. L. R.; Feitosa, T. F; Athayde, A. C. R; Azevedo, S. S; Souto, D. V. O.; Silveira, G. L. da; Melo, L. R. B. de. Sheep gastrointestinal helminthiasis in the Sertão region of Paraíba State, Northeastern Brazil: prevalence and risk factors. Revista B rasileira de Parasitologia Veterinária, v.23, n.4, p.488494, 2014b. http://dx.doi.org/10.1590/S198429612014089 
Vilela, V. L. R.; Feitosa. T. F; Linhares, E. F; Athayde, A. C. R; Molento, M. B; Azevedo, S. S. FAMACHA $\left({ }^{\circ}\right)$ method as an auxiliary strategy in the control of gastrointestinal helminthiasis of dairy goats under semiarid conditions of Northeastern Brazil. Veterinary Parasitology, v. 190, .1-2, p. 281-284, 2012. https://doi.org/10.1016/j.vetpar.2012.05.024 